\title{
The prone position in acute respiratory distress syndrome
}

\author{
Nilay Kocoglu*and Ugur Koca \\ Dokuz Eylul University School of Medicine, Department of Anesthesiology and Reanimation, İntensive Care, Turkey
}

The use of the prone position (PP) during ARDS was studied in 8 randomized controlled trials, 5 of which were larger and 3 smaller. The most recent meta-analysis concluded that there is no statistically significant difference in mortality between the PP group and the supine group. This meta-analysis included 3 sensitivity analyzes on the role of protective ventilation, duration of $\mathrm{PP}$, and the severity of hypoxaemia during inclusion.

When the trial protocol for protective mechanical ventilation was provided, there was no significant reduction in mortality in favor of PP. This reduction in mortality was significant for PP lasting more than 12 hours, but not for shorter PP sessions. The reduction in mortality in favor of PP was significant for most hypoxemic patients with moderate to severe ARDS, but not significant for less hypoxemic patients (mild ARDS) [1].

The PROSEVA study in 27 intensive care units showed a significant reduction in mortality in ARDS patients with $\mathrm{PaO} 2 / \mathrm{FiO} 2$ ratio $(\mathrm{P} / \mathrm{F})<150 \mathrm{mmHg}$ associated with PEEP of at least $5 \mathrm{~cm} \mathrm{H} 2 \mathrm{O}$ after a stabilization period of 12 to 24 hours (at least $60 \%$ FIO2 and tidal volume $6 \mathrm{~mL} / \mathrm{kg}$ PBW). This confirmed a previous meta-analysis on individual data. In the PROSEVA study, patients in the PP group had an average of $4 \mathrm{PP}$ sessions of 17 consecutive hours (protocol planned sessions at least 16 hours). PP continued even without improved oxygenation. PP is inexpensive and easy to apply. It was emphasized that each department should have a written procedure and nursing training for the optimization of PP safety $[2,3]$.

In addition, the documented risks of prone ventilation are conceived in the Berlin definition document that $26.8 \%$ and $31.5 \%$ of patients with mild and moderate ARDS, respectively, should be evaluated with high mortality rates. Even in the recently characterized ARDS subgroup, the rate of rapid recovery that allows extubation within 24 hours is $10.3 \%$. Increased pressure sores or occasional temporary airway obstruction can be seen as acceptable complications in exchange for the possibility of better survival [4].

\section{Prone studies}

1) While no randomized trials have found that prone ventilation reduces mortality in ARDS patients with $\mathrm{P} / \mathrm{F}$ ratios> 150, none of the studies or meta-analyzes are strong enough to do so [5].

2) Two meta-analyzes published before PROSEVA found that prone ventilation had no effect on mortality in medium ARDS, but $70 \%$ of the patients included in these studies received intervention for about 7 hours a day. The next three meta-analyzes found that prone ventilation only reduced mortality when applied $>12 \mathrm{hrs} /$ day [6,7].

3) In a randomized-controlled study by Taccone et al., They looked at the effect of prone ventilation on mortality in patients with moderate and severe ARDS and could not detect a significant benefit of pron in patients with moderate ARDS. In addition to conducting the study with only 192 patients; Taccone and colleagues found no difference in mortality in patients with $\mathrm{P} / \mathrm{F}<100$, unlike the results of PROSEVA. This created concerns about the study [8].

4) A recent observational study shows that prone ventilation is used only in $5.9 \%$ and $10.3 \%$ of mild or moderate ARDS patients and the most common reason for not using prone ventilation is that hypoxemia is not severe enough. Some findings question the idea that the effect of prone ventilation is related to the severity of gas exchange. First, the reduction in mortality reported in Prosave was not associated with the $\mathrm{P} / \mathrm{F}$ ratio, any other measure of oxygenation or carbon dioxide elimination, or changes in gas exchange as a result of proning. A subgroup analysis found that prone ventilation reduced mortality by four quarters of $\mathrm{P} / \mathrm{F}$, but the reduction was higher in patients with $\mathrm{P} / \mathrm{F}$ ratios 105--124 and 124--150 than those with $\mathrm{P}$ / F ratios $<105[9,10]$. Second, Mancebo et al [11] Found that prone ventilation reduced mortality in patients with least severe ARDS with an SAPS II score of $<50$ (including the P / F ratio). No effects were seen in patients with SAPS II> 50.Third, Shen and colleagues recently re-analyzed data from the ARMA study of low tidal volume (VT) ventilation and found that the greatest reduction in mortality was seen in patients with the highest P / F ratios. Among patients ventilated with low VT, the largest mortality difference compared to usual care was seen in those with a P / F of 280. Finally, the metaanalysis by Sud et al. [3] found no evidence that the prone position has a different effect according to the severity of hypoxaemia [11,12].

5) Gattinoni et al argue that only the most severe forms of ARDS "have physiological preconditions for working with prone positioning." However, many studies have shown that prone positioning has beneficial effects on the lung and chest wall. He argued that it served to reduce VILI by reducing excessive distension and airspace collapse in all lungs, including completely normal ones [13-16].

In the newly published American Thoracic Society / European Society of Intensive Care Medicine / Critical Care Medicine Association (ATS / ESICM / SCCM) guidelines, the authors appear to oppose prone ventilation for patients with $\mathrm{P} / \mathrm{F}$ ratios $>100$. This conclusion is derived from the following sentence: "The desired balance is less relied on compared to undesirable consequences."

${ }^{*}$ Correspondence to: Uğur Koca, Dokuz Eylul University School of Medicine, Department of Anesthesiology and Reanimation, İntensive Care, Turkey, Email: ugur.koca@deu.edu.tr

Key words: children, central sleep apnoea

Received: September 07, 2020; Accepted: September 21, 2020; Published: September 28, 2020 
Determining the incidence of adverse outcomes of prone ventilation and whether they occur more frequently than in patients with supine ventilation can be determined by a number of factors. These include: (a) the complications evaluated differ between the various trials; (b) the same complication has been reported to occur at significantly different rates; (c) complication rates in prone ventilated patients were not always compared with rates seen in supine ventilated patients; (d) complication rates may be affected by the degree of sedation, how many times patients are turned each day, and how providers are trained or experienced on how to change patients; (e) centers may differ in the use of interventions designed to prevent complications before they occur. The greatest concern for undesirable results comes from the study where all eight complications monitored by Taccone and colleagues occurred at a significantly higher rate than any other study of prone ventilation. The differences were statistically significant for seven out of eight, again different from other studies in the literature [8].

Taccone et al. Used the Rotoprone bed in most of the prone patients. Rotoprone bed has been associated with poor patient tolerance, increased need for sedation and development of hypotension. This is why Taccone et al. (A) why $80 \%$ of prone patients need increased sedation, but only $50 \%$ of those who are supine, (b) why $72 \%$ of prone patients develop hypotension while others in the literature report the incidence of this complication. explains that three studies developed hypotension, $<33 \%$, and (c) their reported overall complication rate was almost three times higher than those found by the same group of researchers in a previous study that did not use the Rotoprone bed.

The first study by Gattinoni and colleagues on prone ventilation indicated that only half of prone patients needed increased sedation and only $28 \%$ increased muscle relaxants.

In the study by Mancebo et al. [9], the use and dosage of benzodiazepines, opiates, and neuromuscular blocking agents (NMBA) were at the discretion of the treating physicians, with the recommendation that sedation should be titrated to a Ramsay score of 3 \pm 1 . No difference was observed in the cumulative doses of these drugs in prone ventilation versus supine ventilation [11].

Ideally, the depth and duration of sedation should be guided based on standard sedation algorithms, wake-up protocols; not based on body position or severity of ARDS. Recently published meta-analysis of prone ventilation complications found no increased risk of barotrauma, unplanned central catheter removal, unplanned extubations, pneumothorax, cardiac arrest, brady- or tachyarrhythmias, or ventilator-associated pneumonia in prone ventilated patients compared to those who were supine ventilated. The only complications that were more common in prone patients were pressure sores and temporary endotracheal tube obstruction $[11,17]$.

The recently published international prone ventilation observational study was collected from 141 ICUs in 20 countries, and the authors noted that the complication rate was surprisingly low, much lower than the rates reported in previous publications [18]. Using protective covers by determining pressure points, paying attention to the position of the face and using various support surfaces significantly reduce pressure sores. If a staff member is assigned to monitor the endotracheal tube while rotating patients, temporary endotracheal tube occlusion should hardly occur and, if present, should be recognized and quickly and easily reversed, as with published recommendations on how to turn patients. Similarly, if a patient has a thoracostomy tube, it would be prudent to assign responsibility to only one staff member to protect against displacement on return [19].

The benefits of prone ventilation in patients with mild or moderate ARDS are not documented, but neither is useful. Although appropriately designed studies are needed in patients with these less severe ARDS subtypes; It will not allow us to do anything for patients with mild or moderate ARDS other than following current guidelines and recommendations, using low tidal volumes and applying good supportive care as there is no other proven therapy. Given the high mortality rates in these ARDS subtypes, the frequency and severity of possible risks, the chance of clinically significant improvement in survival (based on published confidence intervals), and limiting prone ventilation to patients with $\mathrm{P} / \mathrm{F}$ ratios $<100$ or $<150$ seems overly conservative.

\section{References}

1. Munshi L, Sorbo LD, Adhikari NKJ, Hodgson CL, Wunsch H, et al. (2017) Prone Position for Acute Respiratory Distress Syndrome. A Systematic Review and MetaAnalysis. Ann Am Thorac Soc S280-S288. [Crossref]

2. Guérin C, Reignier J, Richard JC, Beuret P, Gacouin A, et al. (2013) Prone positioning in severe acute respiratory distress syndrome. $N$ Engl J Med 368: 2159-2168. [Crossref]

3. Gattinoni L, Carlesso E, Taccone P, Polli F, Guérin C, et al. (2010) Prone positioning improves survival in severe ARDS: a pathophysiologic review and individual patient meta-analysis. Minerva Anestesiol 76:448-454. [Crossref]

4. Schenck EJ, Oromendia C, Torres KL, Berlin DA, Augustine MK, et al. (2019) Rapidly Improving ARDS in Therapeutic Randomized Controlled Trials. chest 155: 474-482. [Crossref]

5. Bellani G, Laffey JG, Pham T, Fan E, Brochard L, et al. (2016) Epidemiology, Patterns of Care, and Mortality for Patients with Acute Respiratory Distress Syndrome in Intensive Care Units in 50 Countries JAMA 315: 788-800. [Crossref]

6. Sachin S, Friedrich JO, Adhikari NK, Taccone P, Mancebo J, et al. (2014) Effect of prone positioning during mechanical ventilation on mortality among patients with acute respiratory distress syndrome: a systematic review and meta-analysis. CMAJ 186: E381-E390. [Crossref]

7. Beitler JR, Shaefi S, Montesi SB, Devlin A, Loring SH, et al. (2014) Prone positioning reduces mortality from acute respiratory distress syndrome in the low tidal volume era: a meta-analysis. Intensive Care Med 40: 332-341 [Crossref]

8. Taccone P, Pesenti A, Latini R, Polli F, Vagginelli F, et al. (2009) Prone positioning in patients with moderate and severe acute respiratory distress syndrome: a randomized controlled trial. JAMA 302: 1977-1984. [Crossref]

9. Guerin C, Beuret P, Constantin JM, Bellani G, Garcia-Olivares P, et al. (2018) A prospective international observational prevalence study on prone positioning of ARDS patients: the APRONET (ARDS Prone Position Network) study. Intensive Care Med 44: 22-37. [Crossref]

10. Albert RK, Keniston A, Baboi L, Ayzac L, Guérin C, et al. (2014) Prone positioninduced improvement in gas exchange does not predict improved survival in the acute respiratory distress syndrome. Am J Respir Crit Care Med 189: 494-496. [Crossref]

11. Mancebo J, Fernández R, Blanch L, Rialp G, Gordo F, et al. (2006) A multicenter trial of prolonged prone ventilation in severe acute respiratory distress syndrome. $\mathrm{Am} \mathrm{J}$ Respir Crit Care Med 173: 1233-1239. [Crossref]

12. Shen Y, Cai G, Gong S, Dong L, Yan J, et al. (2019) Interaction between low tidal volume ventilation strategy and severity of acute respiratory distress syndrome: a retrospective cohort study. Crit Care 23: 254.

13. Lamm WJ, Graham MM, Albert RK (1994) Mechanism by which the prone position improves oxygenation in acute lung injury. Am J Respir Crit Care Med 150: 184-193. [Crossref]

14. Musch G, Layfield JDH, Harris RS, Melo MFV, Winkler T, et al. (2002) Topographical distribution of pulmonary perfusion and ventilation assessed by PET in supine and prone humans. J Appl Physiol 93: 1841-1851. [Crossref]

15. Henderson AC, Sá RC, Theilmann RJ, Buxton RB, Prisk GK, et al. (2013) The gravitational distribution of ventilation-perfusion ratio is more uniform in prone than supine posture in the normal human lung. J Appl Physiol 115: 313-324. [Crossref] 
16. Del Sorbo L, Goligher EC, McAuley DF, Rubenfeld GD, Brochard LJ, et al. (2017) Mechanical ventilation in adults with acute respiratory distress syndrome. Summary of the experimental evidence for the clinical practice guideline. Ann Am Thorac Soc 14: S261-S270. [Crossref]

17. Lee JM, Bae W, Lee YJ, Cho YJ (2014) The efficacy and safety of prone positional ventilation in acute respiratory distress syndrome: updated study-level meta-analysis of 11 randomized controlled trials. Crit Care Med 42: 1252-1262. [Crossref]
18. Girard R, Baboi L, Ayzac L, Richard JC, Guérin C (2014) The impact of patient positioning on pressure ulcers in patients with severe ARDS: results from a multicenter randomized controlled trial on prone positioning. Intensive Care Med 40: 397-403. [Crossref]

19. Messerole E, Peine P, Wittkopp S, Marini JJ, Albert RK (2002) The pragmatics of prone positioning. Am J Respir Crit Care Med 165: 1359-1363. [Crossref]

Copyright: $(2020$ Kocoglu N. This is an open-access article distributed under the terms of the Creative Commons Attribution License, which permits unrestricted use, distribution, and reproduction in any medium, provided the original author and source are credited. 